\title{
Investigation and Analysis of Nanofluids Thermal Conductivity Models
}

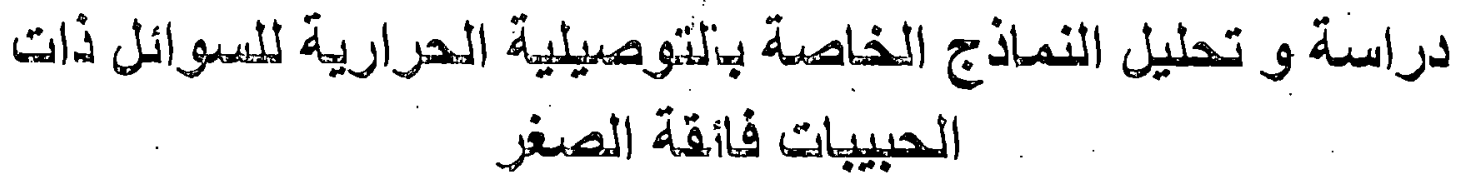

\author{
Mostafa M. Awad*,H. Mar:sour* and H. Abdel-Salam** \\ * Mansoura University, Faculty of Engirnerring, Mech. Power Eng. Dept. \\ ** Dar Al-Handasah Consuitants, Cairo, Egypt.
}

الحدث ألتقدم التقنى فى كثير من التطبيقات الهندسية كالأجهزة الإلكترونية الدقيقة ونقل السمائل ، الحاجة إلى طرق

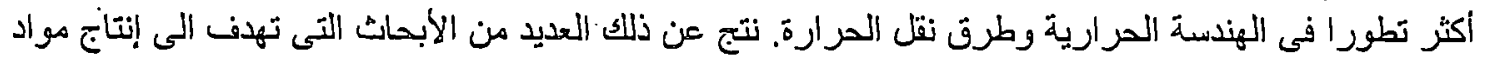

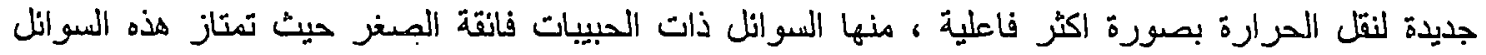
بتوصيلية حرارية عالية متارنة بالسوائل الأساسية كالماء أه الإيثيلين جليكول.

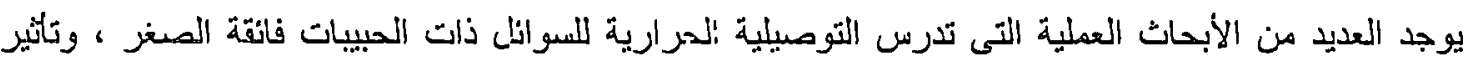

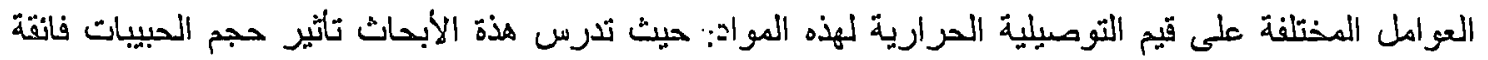

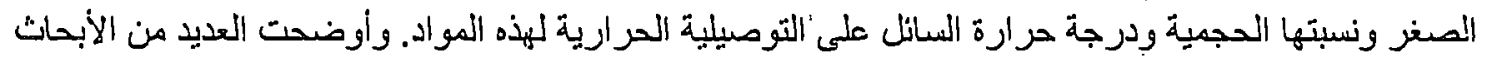

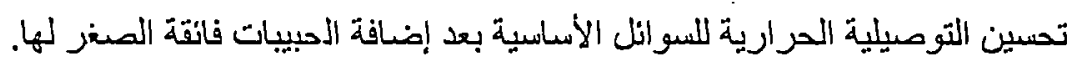

لتفسير هذا التحسن فى التوصيلية الحرارية ، قام العديد من الباحثين بوضع نماذج نظرية للتوصيلية الندرارية

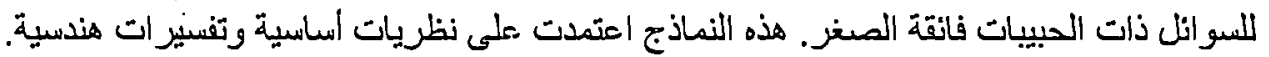

ويهذف هذا البحث المى دراسة وتحليل هذة النماذج لإختبار مدى قابلية كل نموذج للتطبيق فى الظروف العملية

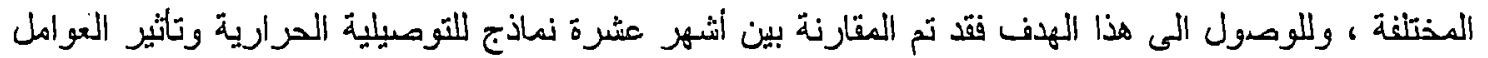

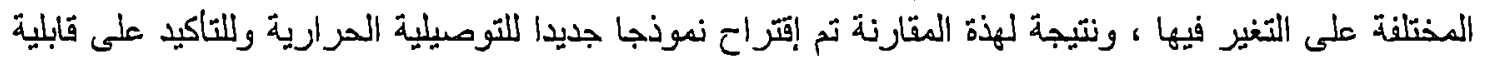

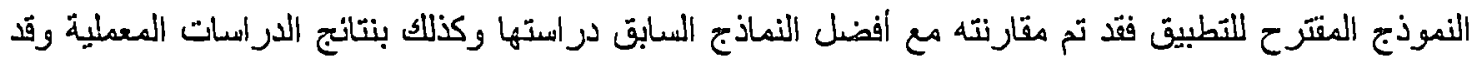

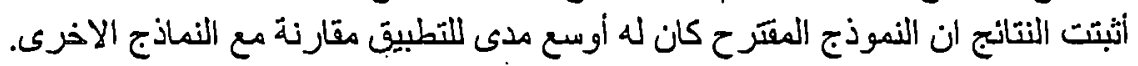

\section{Abstract:}

The thermal conductivity is the key role in presenting the unique thermal properties of nanofluids. The literature involves many theoretical models that i.jvestigate and estimate the thermal conductivity of nanofluids. The main goal of this work is to determine the most applicable model. To achieve this goal, the most famous ten theoretical models have been selected, analyzed and compared with the available experimental data. From this comparison, two models due to Chon et al. [20] and Kumar et al. [23] have been chosen as most applicable models. Based on this analysis and comparison, a new theoretical model dealing with thermal conductivity of nanofluids has been drawn. Testing this proposed model via the available experimental results, shows a more agreement with these results compared with other models. 


\section{Introduction}

Cooling is one of the most important technical challenges facing many diverse industries, including microelectronics, transportation and manufacturing. The conventional methods for increasing heat dissipation are to increase the area available for exchanging heat with a heat transfer fluid as well as increase the flow velocity of the working fluid. However, the former approach requires an undesirable increase in the thermal management system's size and the velocity of the fluid has limited practical values. Furthermore, the inherently poor thermal conductivity of conventional fluids puts a fundamental limit on heat transfer. In contrast, metals have thermal conductivities up to three times higher than these fluids, so it is natural that it would be desired to combine the two substances to produce a heat transfer medium that behaves like a fluid, but has the thermal conductivity of a metal.

A nanofluid is a dilute suspension of nanometer-size particles and fibers that are between 1 and $100 \mathrm{~nm}$ in diameter dispersed in a liquid. As a result, when compared to the base fluid, changes in physical properties of such mixtures occur, e.g., viscosity, density, and thermal conductivity, [1].
The term "Nanofluid", designated for nanoparticle fluid suspension, was first coined by Choi [2] of the Argon National Laboratory, USA in 1995. Nanofluids typically employ metal or metal oxide nanoparticles, such as copper $(\mathrm{Cu})$, aluminum oxide-alumina $\left(\mathrm{Al}_{2} \mathrm{O}_{3}\right)$, copper oxide $(\mathrm{CuO})$, gold $(\mathrm{Ag})$, silicon carbide ( $\mathrm{SiC}$ ), titanium carbide ( $\mathrm{TiC}$ ), titanium oxide $\left(\mathrm{TiO}_{2}\right)$ and carbon nanotubes. The most common nanoparticles are $\mathrm{Al}_{2} \mathrm{O}_{3}$ and $\mathrm{CuO}$. The base fluid is usually a conductive fluid, such as water, ethylene glycol or engine oil. Nanofluids commonly contain up to a $5 \%$ volume fraction of nanoparticles to obtain effective heat transfer enhancements.

Of all the physical properties of nanofluids, the thermal conductivity $\left(k_{n f}\right)$ is the most complex and for many applications the most important one. Interestingly, experimental findings have different results and theories do not fully explain the mechanisms of elevated thermal conductivity.

Recent theoretical models for nanofluid thermal conductivity are presented and compared. Concerning theories/correlations which try to explain thermal conductivity enhancement for all nanofluids, int a single model can predict a wide range of experimental 
data. However, many experimental data isets may: fit between the lower and upper mean-field bounds originally proposed by Maxwell where the static nanoparticle: configurations may range from a dispersed phase to a pseudo-continuous: phase:

ai "Dynamic models, assuming noninteracting metallic nano-spheres, postilate an enhancement above the classical Maxwell theory and thereby provide potentially additional physical insight. Clearly, it will be necessary to consider not only one possible mechanism but combine severai mechanisms and compare predictive results to new benchmark experimental data sets.

In the present work, experimental results and the theoretical studies are reviewed for nanofluid thermal conductivity, with the objective of finding the best models with agree enough with the experimental results and propose a new model that may be in a good agreement with all experimental data considering the effect of particles volume fraction, particle size and base fluid temperature.

\section{Theoretical Studies}

The present models stand for understanding thermal transport, and thus thermal conductivity of nanofluids can be grouped into two categories. The first category groups have started from the nanostructure of nanofluids; assume that a nanofluid is a mixture consisting of a continuous base fluid component called a matrix and a discontinuous solid component called particles. The properties of nanofluids depend on the details of their microstructures, such as the component properties', component volume concentrations; '. particle dimension, particle geometry; particle distribution, $\cdots$ and matrix-particle interfacial effects, $[2,3]$.

Many studies have been conducted using this approach, such as Maxwell [4], Hamilton-Crosser [5], Bruggeman [6], $X u e$ and $\mathrm{Xu}$ [7] and the so-called effective medium theory, [8]. The other category postulate that the thermal conductivity of nanofluids is composed of the particle's conventional static part and a Brownian motion part which produces micro-mixing. These models most referenced as dynamic models. These models take the particle dynamics into consideration, whose effect is additive to the thermal conductivity of a static dilute suspension. Thus, the particle size, volume fraction, thermal conductivities of both the nanoparticle and the base .uid, and the temperature are taken into account in such models for 
the thermal conductivity of nanofluids, [3].

The first dynamic model was proposed by Wang et al. [9]. The models categorize in this type include Xuan [10], Jang \& Choi [11, 12], Prasher [13], Koo \& Kleinstreuer [14] and Vasu et al [15].

\subsection{Classical models}

More than a century ago, Maxwell [4] derived an equation for calculating the effective thermal conductivity of solid-liquid mixtures consisting of spherical particles:

$$
\mathrm{K}_{\mathrm{eff}}=\left(\frac{k_{p}+2 k_{f}+2\left(k_{p}-k_{f}\right) \emptyset}{k_{p}+2 k_{f}-\left(k_{p}-k_{f}\right) \emptyset}\right)
$$

where $\cdot K_{\text {eff }}$ is the effective thermal conductivity of nanofluid normalized to the thermal conductivity of the base fluid, $k_{p}$ is the thermal conductivity of the nanoparticle, $k_{f}$ is the thermal conductivity of the base fluid and $\emptyset$ is the nanoparticles volume fraction, [3].

As seen from this expression, the effect of the size and shape of the particles was not included in the analysis. It should be noted that the interaction between the particles was also neglected in the derivation.

Hamilton and Crosser [5] extended the Maxwell model in order to take the effect of the shape of the solid particles into account, in addition to the thermal conductivities of solid and liquid phases and particle volume fraction. The model is as follows:

$K_{e f f}=\left(\frac{k_{p}+(n-1) k_{f}-(n-1)\left(k_{p}-k_{f}\right) \emptyset}{k_{p}+(n-1) k_{f}+\left(k_{p}-k_{f}\right) \emptyset}\right)$

where $\mathrm{n}$ is the empirical shape factor given by $\mathrm{n}=3 / \psi$, and $\psi$ is the particle sphericity, defined as the ratio of the surface area of a sphere with volume equal to that of the particle, to the surface area of the particle (For example, $n=3$ for spheres and $n=6$ for cylinders). Comparison of $\mathrm{H}-\mathrm{C}$ model to Maxwell model reveals that Maxwell's model is a special case of $\mathrm{H}-\mathrm{C}$ model for sphericity equal to one.

Both Maxwell and Hamilton and Crosser models were originally derived for relatively larger solid particles that have diameters on the order of millimeters or micrometers. Therefore, it is questionable whether these models are able to predict the effective thermal conductivity of nanofluids. Nevertheless, these models are utilized frequently due to their simplicity in the study of nanofluids to have a comparison between theoretical and experimental findings.

Bruggeman [6] proposed a model to analyze the interactions among randomly distributed spherical particles, Bruggeman mulel gives: 
$\varnothing\left(\frac{k_{p}-k_{e f f}}{k_{p}+2 k_{e f f}}\right)+(1-\emptyset)\left(\frac{k_{p}-k_{e f f}}{k_{p}+2 k_{e f f}}\right)=0$

for low solid concentrations, the Bruggeman model results in almost the same results as the Maxwell model, [3].

Effective medium theory, [8] is an improvement of Maxwell model which implement effect of the liquid layer formed on nanoparticle surfaces and its impact on the particle thermal conductivity and on the effective particle volume fraction. The model was developed by Schwartz et al. [16], and was expressed as:

$K_{e f f}=\left(\frac{k_{c p}+2 k_{f}+2\left(k_{c p}-k_{f}\right) \emptyset_{e f f}}{k_{c p}+2 k_{f}-\left(k_{c p}-k_{f}\right) \emptyset_{e f f}}\right) k_{f}$

where $k_{c p}$ is the equivalent thermal conductivity of the "composite nanoparticles", $\mathrm{k}_{\mathrm{cp}}$, can be expressed as:

$$
K_{c p}=\frac{\left[2(1-\alpha)+(1+\xi)^{3}(1+2 \alpha)\right] \alpha}{-(1-\alpha)+(1+\xi)^{3}(1+2 \alpha)} k_{p}
$$

where $\alpha=k_{\text {layer }} / k_{p}$, is the ratio of nanolayer's and particle's thermal conductivities; $\xi=\mathrm{h} / \mathrm{r}$ is the ratio of the ordered layer thickness to the radius of the nanoparticle, and $\varnothing_{\text {eff }}$ is the effective volume of nanoparticles $\left(\emptyset_{\text {eff }}=\right.$ $\left.\emptyset(1+\xi)^{3}\right)$ increases due to the formation of nanolayers.

$\mathrm{Xue}$ and $\mathrm{Xu}$ [7] obtained an equation for the effective thermal conductivity according to Bruggeman model. Their model takes account of the effect of interfacial shells by replacing the thermal conductivity of nanoparticles with the assumed thermal conductivity of the so-called "complex nanoparticles", which included the interfacial shells between the nanoparticles and the base fluids.

$\left(1-\frac{\emptyset}{\alpha}\right) \frac{k_{e f f}-k_{f}}{2 k_{e f f}+k_{f}}+$

$\frac{\emptyset}{\alpha} \frac{\left(k_{e f f}-k_{2}\right)\left(2 k_{2}+k_{1}\right)-\alpha\left(k_{1}-k_{2}\right)\left(2 k_{2}+k_{e f f}\right)}{\left(2 k_{e f f}+k_{2}\right)\left(2 k_{2}+\dot{1}_{1}\right)+2 \alpha\left(k_{1}-k_{2}\right)\left(k_{2}-k_{e f f}\right)}=0$

where $\alpha$ is the volume ratio of spherical nanoparticle and complex nanoparticle. $k_{1}$ and $k_{2}$ are the thermal conductivity of the nanoparticle and interfacial shell, respectively. The modified model is in good agreement with the experimental data on the effective thermal conductivity of $\mathrm{CuO} /$ water and $\mathrm{CuO} / \mathrm{EG}$ nanofluids.

\subsection{Dynamic models}

Xuan et al. [10] developed a dynamic model into which the effects of Brownian motion of nanoparticles and the aggregation structure of nanoparticle clusters (i.e., fractals) are taken. Their model is expressed as:

$$
\begin{gathered}
K_{e f f}=\frac{k_{p}+2 k_{f}-2\left(k_{f}-k_{p}\right) \emptyset}{k_{p}+2 k_{f}+\left(k_{f}-k_{p}\right) \emptyset} k_{f}+ \\
\frac{\rho_{p} \emptyset c_{p}}{2} \sqrt{\frac{k_{B} T}{3 \pi r_{c} \mu_{f}}}
\end{gathered}
$$

where Boltzmann constant $\mathrm{k}_{B}=1.381 \times$ $10^{-23} \mathrm{~J} / \mathrm{K}, \mathrm{r}_{C}$ is the apparent radius of 


\section{122 Mostafa M. Awad, H. Mansour and H. Abdel-Salam}

clusters and depends on the fractal dimension of the cluster structure, $T^{\prime}$ is the fluid temperature, $\mu_{f}$ is the dynamic viscosity of the base fluid, $\rho_{p}$ is the particle density and $c_{p}$ is the specific heat of nanoparticle material.

Although this model incorporates the effect of temperature on the thermal conductivity enhancement, the dependence is too weak $\left(\alpha T^{1 / 2}\right)$ and not in agreement with the experimental data of Das et al. [17].

Kumar et al. [18] involves the combination of the stationary particle model and the moving particle model. The stationary particle model looks at the increased surface area as the particle size decreases. By assuming two parallel paths of heat flow (one through base fluid molecules and the other through the nanoparticles), this model shows the linear dependence of thermal conductivity on particle concentration and the inverse dependence of thermal conductivity on the size of the particle. The effective thermal conductivity of the nanofluids, $K_{e f f}$, for this model is given by:

$K_{e f f}=k_{f}+c \frac{2 k_{B} T}{\pi v d_{p}^{2}} \frac{\emptyset r_{f}}{k_{f}(1-\emptyset) r_{p}} k_{f}$

where $\mathrm{c}$ is a constant, $\mathrm{r}_{f}, \mathrm{r}_{p}$ and $v$ are the radius of the base fluid molecules, the radius of the nanoparticles, and the kinematic viscosity of the base fluid respectively. However, the validity of the model has got to be established; it may not be suitable for high concentration of particles and for nanoparticles with radius larger than that of the liquid molecules, [19].

Jang and Choi $[11,12]$ devised a theoretical model that involves four modes such as collision between base fluid molecules, thermal diffusion in nanoparticles in fluids, collision between nanoparticles due to Brownian motion, and thermal interaction of dynamic nanoparticles with the base fluid molecules. The resulting expression for the effective thermal conductivity of nanofluids is:

$$
\begin{gathered}
K_{e f f}=k_{f}(1-\emptyset)+k_{p} \emptyset+ \\
3 C \frac{d_{f}}{d_{p}} k_{f} \operatorname{Re}_{d_{p}}^{2} \operatorname{Pr} \emptyset
\end{gathered}
$$

where $\mathrm{C}$ is a proportional constant equals to $18 * 10^{6}, \mathrm{Pr}$ is the Prandtl number defined as $\operatorname{Pr}=\frac{\mu_{f} \mathrm{Cp}}{k_{f}}$ and $\operatorname{Re}_{\mathrm{dp}}$ is Reynolds number defined as $\mathrm{Re}_{\mathrm{dp}}=$ $\frac{C_{\mathrm{RM}} d_{p}}{v_{f}}$, where $\mathrm{C}_{\mathrm{RM}}$ is the random motion velocity of the base fluid defined as $C_{\mathrm{RM}}=\frac{D_{0}}{L_{\mathrm{BF}}}, \mathrm{D}_{0}$ is the nanoparticle diffusion coefficient defined as $D_{0}=$ $\frac{k_{B} T}{3 \pi \mu_{f} d_{p}}$, and $\mathrm{L}_{B F}$ is the mean free path of 
the base fluid molecule, and it is $0.17 \mathrm{~nm}$ for water.

The advantage of the model is to include the effects of concentration; temperature, and particle size. However, the Brownian effect was neglected, which may not be suitable since the high temperature dependent properties may caused by the Brownian motion.

Koo and Kleinstreuer [14] developed a new model for nanofluids, which includes the effects of particle size, particle volume fraction and temperature dependence as well as properties of the base fluid and the particle subject to Brownian motion. The resulting formula is:

$$
\begin{aligned}
K_{c f f}= & \frac{k_{p}+2 k_{f}+2\left(k_{p}-k_{f}\right) \emptyset}{k_{p}+2 k_{f}-\left(k_{p}-k_{f}\right) \emptyset} k_{f}+ \\
& 5 * 10^{4} \beta \emptyset \rho_{p} c_{p} \sqrt{\frac{k_{B} T}{\rho_{p} D}} f(T, \emptyset)
\end{aligned}
$$

Note that the first part of Eq. (10) is obtained directly from the Maxwell model while the second part accounts for Brownian motion, which causes the temperature dependence of the effective thermal conductivity. $f(T, \varnothing)$ can be assumed to vary continuously with the particle volume fraction,

$f(T, \varnothing)=(-6.04 \varnothing+0.4705) T+(1722.3 \varnothing-134.63)$

while $\beta$ is related to particle motion.
Chon et al. [20] investigated the thermal conductivity of $\mathrm{Al}_{2} \mathrm{O}_{3}$ /water nanofluid experimentally and proposed a correlation for the determination of the thermal conductivity of $\mathrm{Al}_{2} \mathrm{O}_{3}$ nanofluids based on the experimental data. The correlation provided is:

$$
\begin{aligned}
K_{e f f}= & k_{f}+64.7 \phi^{0.746}\left(\frac{d_{f}}{d_{p}}\right)^{0.369}\left(\frac{k_{p}}{k_{f}}\right)^{0.7476} * \\
& \operatorname{Re}_{\mathrm{dp}}{ }^{1.2321} \mathrm{Pr}^{0.9955} \mathrm{~K}_{\mathrm{f}}
\end{aligned}
$$

where $d_{f}$ is the diameter of the fluid molecules. Prandtl number and Reynolds number are defined as:

$$
\begin{gathered}
\operatorname{Pr}=\frac{\mu_{f}}{\rho_{f} \alpha_{f}} \\
\operatorname{Re}=\frac{\rho_{f} V_{\mathrm{Br}} d_{p}}{\mu_{f}}
\end{gathered}
$$

where $\alpha_{f}$ is the thermal diffusivity of the base fluid. $\mathrm{V}_{B r}$ is the Brownian velocity of the nanoparticles and it is calculated using the following expression:

$$
V_{\mathrm{Br}}=\frac{k_{B} T}{3 \pi \mu_{f} d_{p} \lambda_{f}}
$$

Where $k_{B}$ is Boltzmann constant and $T$ is temperature in $\mathrm{K} . \lambda_{\mathrm{f}}$ is mean-free path of the fluid molecules. The validity range of the correlation is between $11 \mathrm{~nm}$ and 150 $\mathrm{nm}$ for particle diameter, $1 \%$ and $4 \%$ for particle volume fraction, and $21^{\circ} \mathrm{C}$ and $71^{\circ} \mathrm{C}$ for temperature.

Moghadassi et al. [21] presents a novel model for the prediction of the 
effective thermal conductivity of nanofluids based on dinensionless groups. The model expresses the thermal conductivity of a nanofluid as a function of the thermal conductivity of the solid and liquid, their volume fractions, particle size and interfacial shell properties. According to this model, thermal conductivity changes nonlinearly with nanoparticle loading.

$$
K_{e f f}=k_{f}+m \frac{(\phi)^{\alpha}}{\left(d_{p}\right)^{B}} k_{f}
$$

where $m$ is a factor that depends on the properties of the solid particle and interfacial shell, while $\alpha$ and $\beta$ are empirical constants determined from experimental data.

Morajevi et al [22] proposed a new model for the thermal conductivity of $\mathrm{Al}_{2} \mathrm{O}_{3}+\mathrm{H}_{2} \mathrm{O}$ and $\mathrm{CuO}+\mathrm{H}_{2} \mathrm{O}$ nanofluid mixtures considering the volume fraction and shape factor of nanoparticles.

$$
K_{e f f}=\frac{\frac{4.1 k_{p} k_{f} \phi}{3.1 k_{f}+k_{p}}+k_{f}(1-\phi)}{\phi\left(\frac{4.1 k_{f}}{3.1 k_{f}+k_{p}}-1\right)+1}
$$

The derivation of the model relied on experimental images to nanoparticles shape, which indicated that the nanoparticles are not complete spherical particles. Therefore, shape factors between 3 (spherical) and 4.5 (middle of spherical and cylindrical assumption of particle) was considered. Then by fitting the empirical data, 4.1 was selected.

Kumar et al. [23] proposed a new thermal conductivity model is proposed based on the combination of statistical mechanism model and Brownian motion with the inclusion of particle critical size.

$$
\begin{aligned}
\mathrm{K}_{\mathrm{eff}}= & \frac{\mathrm{k}_{\mathrm{p}}+\left(\mathrm{n}_{\mathrm{s}}-1\right) \mathrm{k}_{\mathrm{f}}-\left(\mathrm{n}_{\mathrm{s}}-1\right) \phi_{\text {eff }}\left(\mathrm{k}_{\mathrm{f}}-\mathrm{k}_{\mathrm{p}}\right)}{\mathrm{k}_{\mathrm{p}}+\left(\mathrm{n}_{\mathrm{s}}-1\right) \mathrm{k}_{\mathrm{f}}+\phi_{\text {eff }}\left(\mathrm{k}_{\mathrm{f}}-\mathrm{k}_{\mathrm{p}}\right)}+ \\
& \frac{C 1 \phi_{e f f}\left(T-T_{0}\right)}{\mu_{f} k_{f} \tau_{p}^{4}}-
\end{aligned}
$$

where the value of constant $\mathrm{C}$ and temperature $T_{0}$ are set to equal $7 \times 10^{-36}$ and $21^{\circ} \mathrm{C}$ respectively.

This model is compared with $\mathrm{Al}_{2} \mathrm{O}_{3} /$ water and $\mathrm{CuO} /$ water based nanofluids of spherical particles using the well-known thermal conductivity models and the experimental results available in the open literature. This model is found fits well with the existing Brownian motion theoretical model and experimental results. It concludes that thermal conductivity is enhanced due to the effect of shape, nanolayer and Brownian motion of the particles. The Brownian motion contribution is significant only when the particle size is less than that of critical size and optimum particle volume fraction.

\section{Comparison with Experimental}

Data 
Theoretical models were presented above, root from different theories, investigations and assumptions. To perform a precise comparison, detect models pros and cons, and obtain qualitative differences between the models, specific comparisons between models versus published experimental results whose parameters are identical or. have very closed values, will be configured.

The experimental studies performed by; Das et al. [17], Beck [24], Minsta et al. [25] and Patel et al. [26] are used as the base of comparison. In these studies the used nanoparticle was $\mathrm{Al}_{2} \mathrm{O}_{3}$ with sizes $38.4 \mathrm{~nm}, 46 \mathrm{~nm}, 47 \mathrm{~nm}$ and 45 nm used by Das, Beck, Minsta and Patel, respectively. Nanoparticle concentrations in the experiments were $3 \%$ and $4 \%$ by volume. The experiments were done at the room temperature $\left(20-21^{\circ} \mathrm{C}\right)$.

From the theoretical and empirical models reviewed in the previous section, ten models are selected for such comparison; Maxwell model [4], Effective medium theory [8], Xuan [10], Jang and Choi [11, 12], Kumar [18], Koo and Kleinstreuer [14], Chon [20],
Moghadassi [21], Morajevi [22] and Kumar [23]. These models cover the most theories and improvement mechanisms that ..explaining the nanofluids thermal conductivity enhancements.

In order to examine the theoretical models, comparisons of the experimental results versus theoretical models based on the previously mentioned parameters will be shown. In the following sections, experimental studies about the thermal conductivity of nanofluids are summarized. In each section, a specific parameter that affects the nanofluids thermal conductivity is discussed.

\subsection{Effect of particle volume fraction}

Figure (1) shows the effect of nanoparticle volume fraction on the thermal conductivity enhancement of $\mathrm{Al}_{2} \mathrm{O}_{3}$ /water nanofluid. Ten theoretical models are considered in addition to the available experimental data of Patel et al. [26], Beck [24] and Das et al. [17]. These experimental studied were performed on 45,46 and $38 \mathrm{~nm}$ respectively and at room temperature. 
M. 126 Mostafa M. Awad, H. Mansour and H. Abdel-Salam

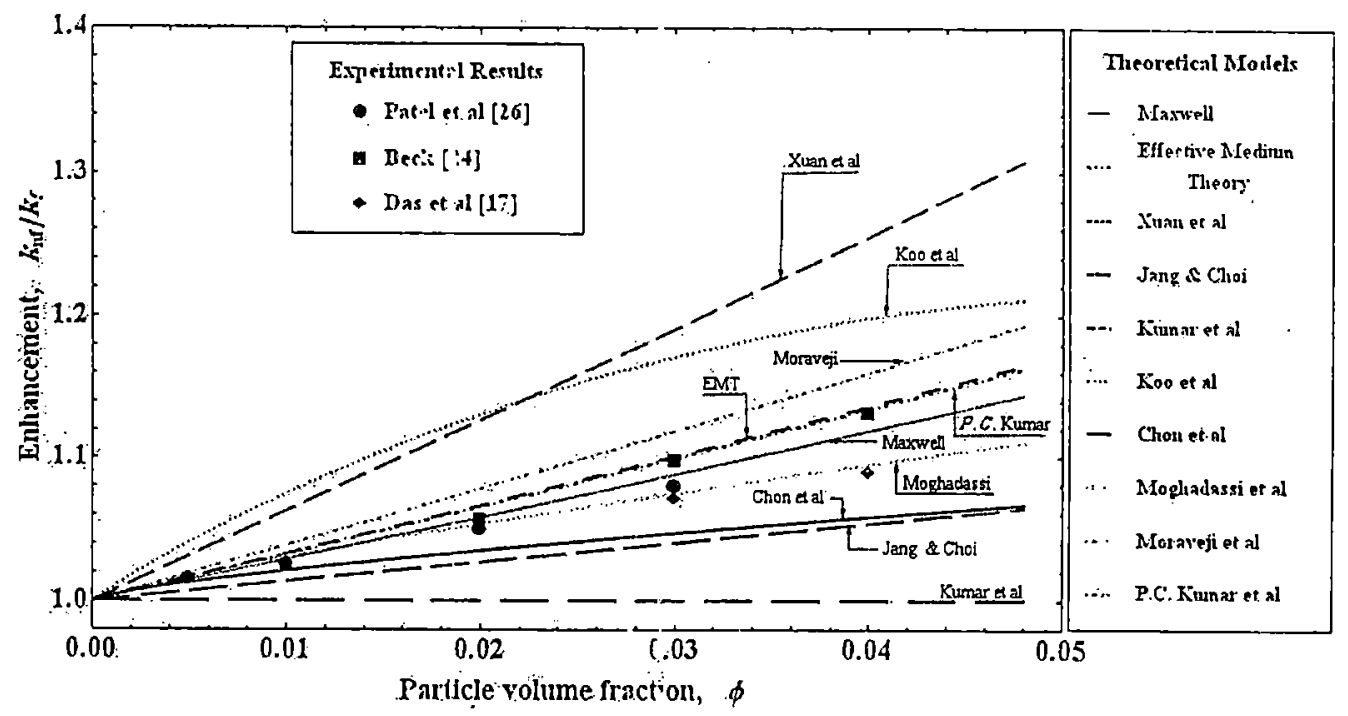

Fig. 1 Effect of nancparticle volurie fraction on thermal conductivity enhancement of $\mathrm{Al}_{2} \mathrm{O}_{3}$ /water nanofluid.

Out of these curves Maxwell, Effective medium theory, Moghadassi, Chon and P.C. Kumar show good agreement with experimental results.

\subsection{Effect of particle size:}

The results of experimental works based on of $\mathrm{Al}_{2} \mathrm{O}_{3}$ /water nanofluid with volume fraction of $3 \%$ at room temperature, are illustrated on Fig. 2.

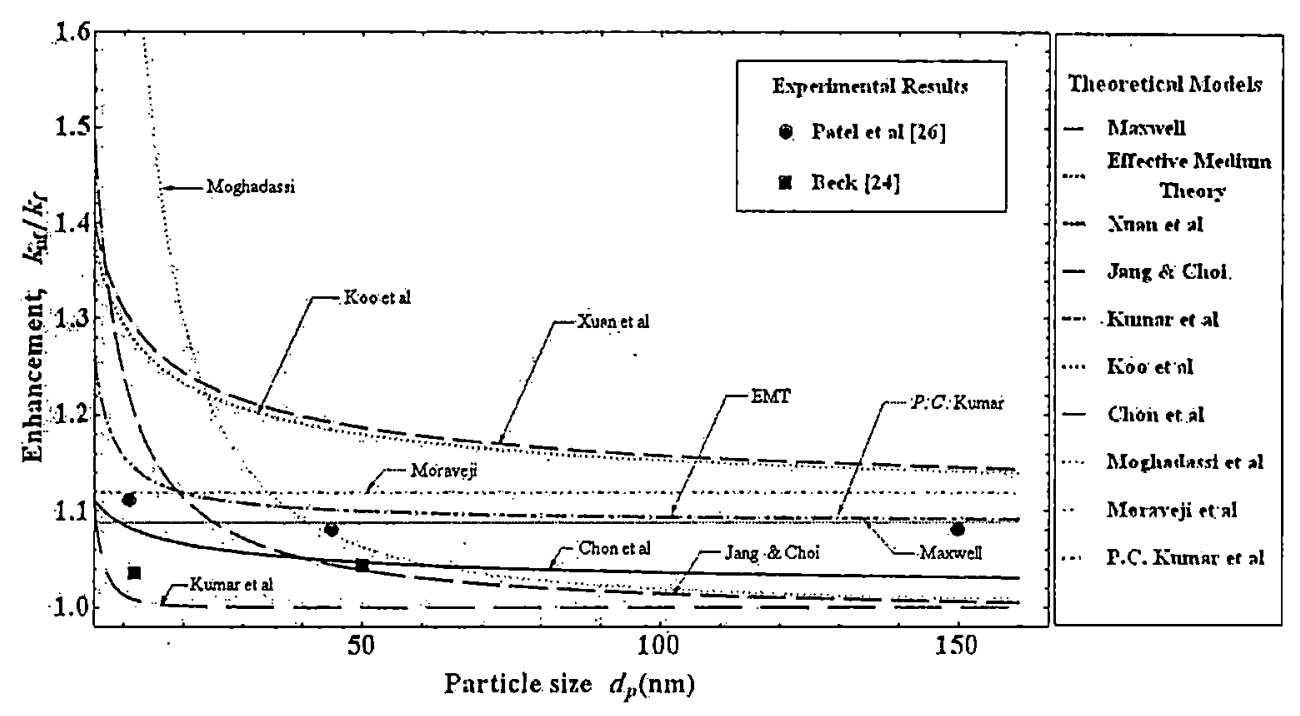

Fig. 2. Effect of nanoparticle size on thermal conductivity enhancement of $\mathrm{Al}_{2} \mathrm{O}_{3}$ /water nanofluid

Out of these curves, Jang-Choi, Kumar, Moghadassi, Chon et al. and P.C. Kumar show good agreement with experimental results. Models of Maxwell and Xuan et al. show no changes with particle size. 


\subsection{Effect of base fluid teraperature}

The effect of temperature change of nanofluids base fluid temperature on the enhancement of nanofluid; thermal conductivity is shown in Fig. (4). A comparison between the different models: with the experimental results performed at a volume fraction of $3 \%$ indicates thâ.
Jang-Choi, Chon et al and P.C.Kumar show good agreement with experimental results. Koo et al. curve is overestimated the experimental results, while Maxwell, Kumar, EMT and Xuan et al show no changes temperature change

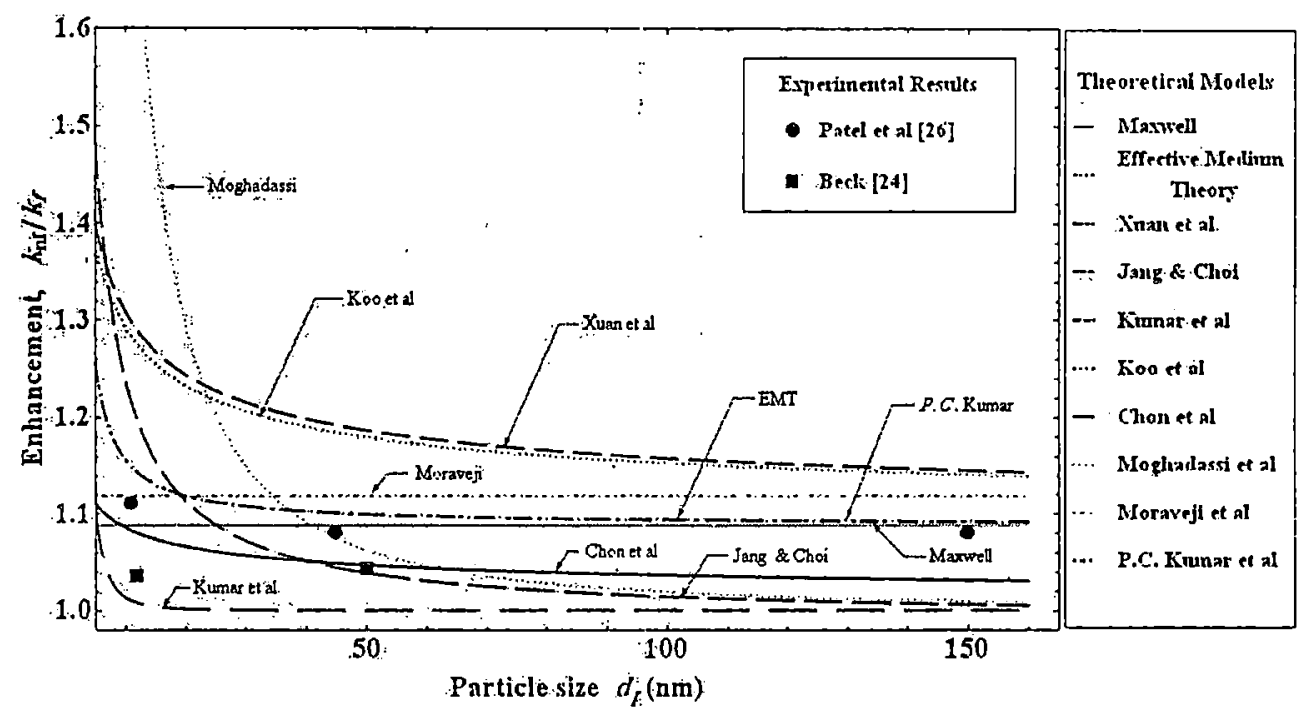

Fig. 3 Effect of base fluid temperature on thermal conductivity enhancement of $\mathrm{A}_{2}^{\prime} \mathrm{O}_{3}$ /water nanofluid

\section{Results and Discussions:}

It is clear from the above comparisons that each theoretical model is suitable to describe the nanofluid. thermal conductivity for a specific case of comparison and at the same time does not agree enough with the other cases.

From figures.1, 2, and 3, it can be noted that the theoretical models due to Chon et al. [20] and Kumar et al. [23] are in general agree enough with the experimental data for the ail cases of comparisons. It means that these two models are valid to describe the behavior of nanofluids at different volume fractions, different particle sizes and different base fluid temperature. Figures 4, 5 and 6 illustrate the above remarks.

Also it can be noticed that the experimental results of Patel et al. [26] are shown to take the mean values among the other experimental results for all cases of comparisons. 


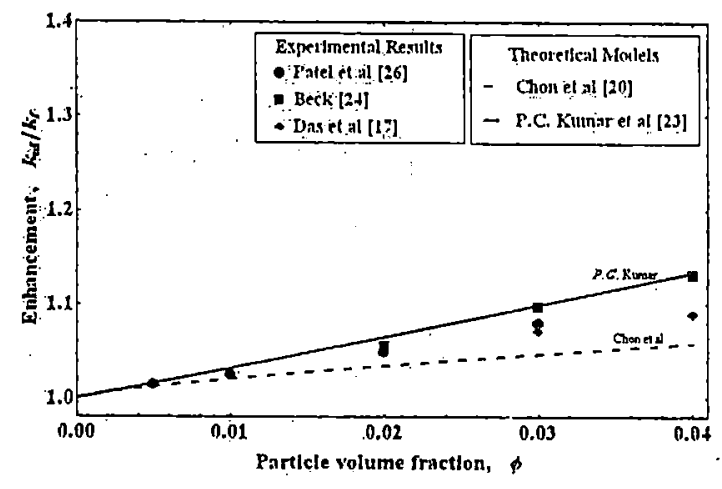

Fig. 4 Effect of nanoparticle volume fraction on thermal conductivity enhancement of $\mathrm{Al}_{2} \mathrm{O}_{3}$ /water nanofluid (Chon and Kumar models)

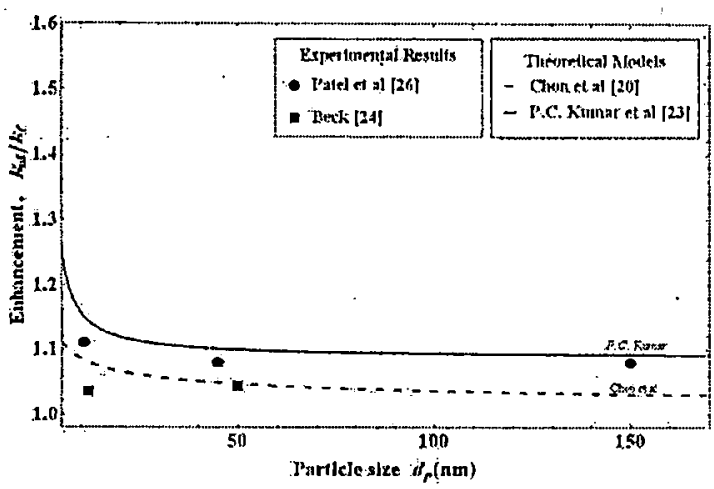

Fig. 5 Effect of nanoparticle size on thermal conductivity enhancement of $\mathrm{Al}_{2} \mathrm{O}_{3}$ /water nanofluid (Chon and Kumar models)

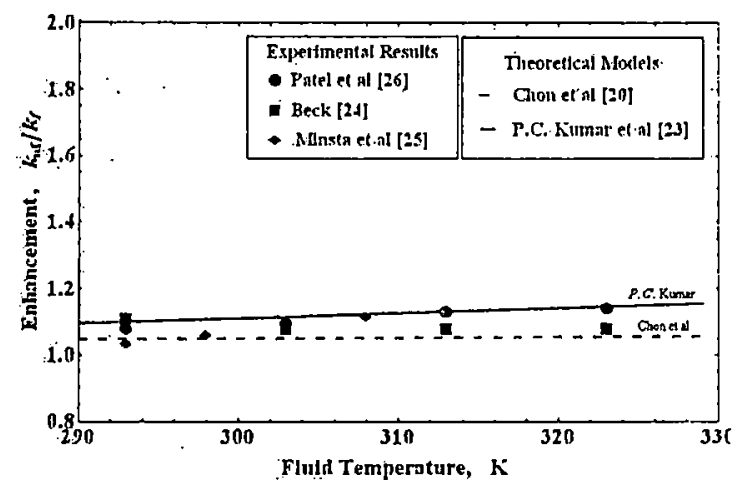

Fig. 6 Effect of base fluid temperature on thermal conductivity enhancement of $\mathrm{Al}_{2} \mathrm{O}_{3}$ /water nanofluid (Chon and Kumar models)

5. Proposed Model for the Thermal Conductivity of Nanofluids
As mentioned above, the thermal conductivity of nanofluids is afferted by many parameters. Among of these parameters are the particle size, the particle volume fraction and the base fluid temperature. But none of the proposed models is suitable to describe the nanofluids behavior, when considering all the effective parameters with each other. Therefore, one of the objectives of this study is to propose a new model, which can be able to describe the nanofluids behavior for a wide range of the various mentioned parameters.

The above comparisons concluded that Chon et al. model [20] and P.C. Kumar et al. model [23] are the most suitable models to predict the nanofluids thermal conductivity over various parameters. Chon et al. model [20] is shown in correlation (11).

The model correlation contain two terms. The first term is the base fluid thermal conductivity. The second term contains the "enhancement part" of the correlation. The second term will be diminished if the particle volume fraction turns to zero, also the term contains dimensionless groups with powers determined empirically.

The proposed model utilizes the "enhancement term" of Chon model with a new value of empirical constant $C$ to fit 
the experititiental data. Jang and Choi model $[11,12]$ with its modification by Izadi et al. [27] for interfacial. resistance at solid particle interface gives good agreement with experimental results especially when investigating the particle size effect. The proposed model shown in correlation (15) utilizes the advantages detected in these two models as follows;

$$
\begin{gathered}
K_{e f f}=k_{f}(1-\phi)+\beta_{K R} k_{p} \phi_{e f f}+ \\
C \phi_{e f f}{ }^{0.746}\left(\frac{d f}{d_{p}}\right)^{0.369}\left(\frac{k_{p}}{k_{f}}\right)^{0.7476} * \\
\operatorname{Re}_{d p}{ }^{1.2321} \operatorname{Pr}^{0.9955} k_{f}
\end{gathered}
$$

where $C$ is an empirical constant equals 125 and $\beta_{K R}$ represent the thermal interfacial resistance equals $4 * 10^{-8}$. The first two terms of the model are extracted from Jang and Choi (and its modification by Izadi et al.) model.

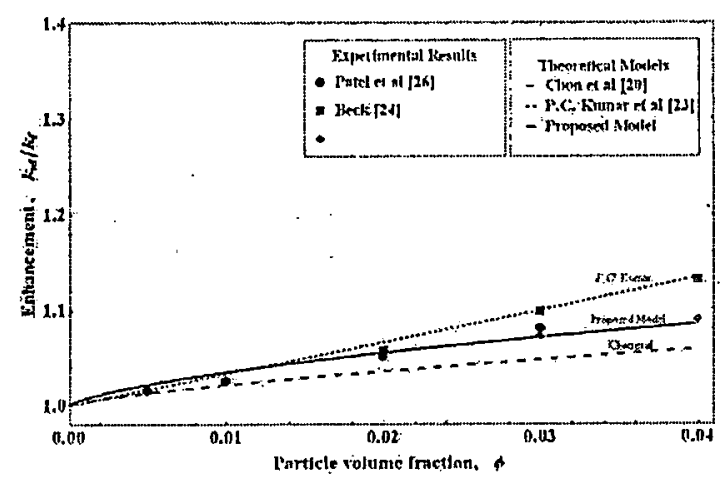

Fig. 7 Effect of nanoparticle volume fraction on thermal conductivity enhancement of $\mathrm{Al}_{2} \mathrm{O}_{3}$ /water nanofluid (Chon, Kumar and proposed models)
These terms contribute the effects of collision of base fluid molecules and thermal diffusion in nanoparticles in fluids, which physically indicates the heat transfer by thermal conductivity of nanoparticles, [12]. The last term is extracted from Chon et al model. the term implement the effects of nanoparticles Brownian motion. The empirical constant, $C$, value is modified to be 125 to fit the experimental data results for $\mathrm{Al}_{2} \mathrm{O}_{3}$.

Comparisons of the proposed model with the experimental results and with Chon et al. and Kumar et al. models are presented in Figures 7, 8 and 9 for particle volume fraction effect, particle size effect and base fluid temperature effect respectively.

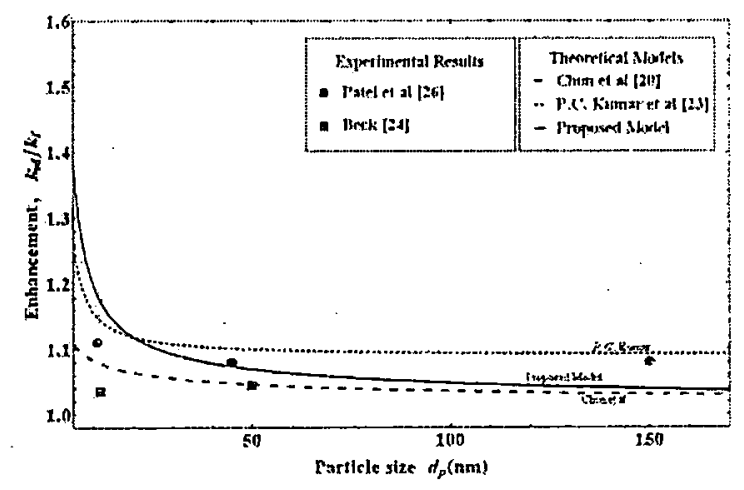

Fig. 8 Effect of nanoparticle size on thermal conductivity enhancement of $\mathrm{Al}_{2} \mathrm{O}_{3}$ /water nanofluid (Chon, Kumar and proposed models) 


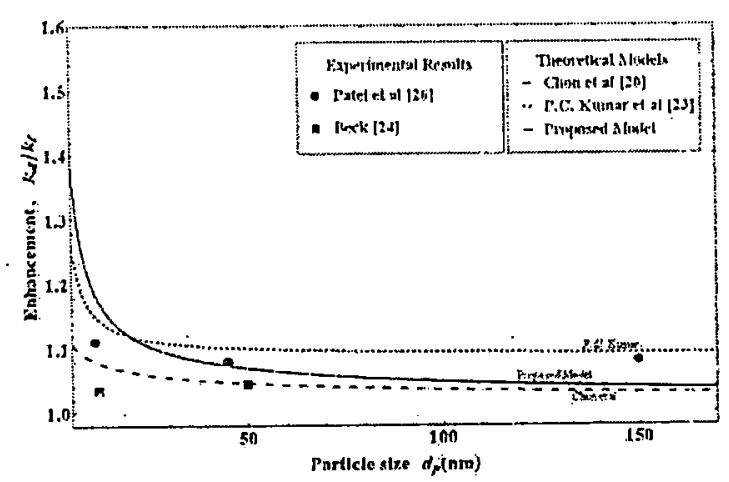

Fig. 9 Effect of base fluid temperature on thermal conductivity enhancement of $\mathrm{Al}_{2} \mathrm{O}_{3}$ /water nanofluid (Chon: Kumar proposed models)

Figures 7,8 and 9 show that, the proposed model is in a good agreement with experimental results for a wide range of the most effective parameters; volume fraction, nanoparticle size and base fluid temperature.

\section{Conclusions}

The investigation of nanofluids thermal conductivity throughout the current study concluded the following:

1. Comparison among ten proposed models and three experimental works indicated that some models of nanofluids thermal conductivity show good agreement with experimental results for changes in some compared parameter while not for other parameters.

2. Models due to Maxwell, Effective medium theory, Moghadassi, Chon and Kumar show good agreement with - experimental results for changes in nanoparticle volume fractions.

3. Models due to Jang-Choi, Kumar, Moghadassi, Chon et al and P.C. Kumar show good agreement with experimental results for different particle sizes.

4. Models due to Jang-Choi, Chon et al and P.C.Kumar show good agreement with experimental results for changes in base fluid temperature.

5. Models due to Chon et al [20] and Kumar et al [23] are in general agree enough with the experimental data for the changes of nanoparticle volume fraction, nanoparticle size and base fluid temperature.

6. A new model was proposed to describe the thermal conductivity of nanofluids, which is suitable to predict the thermal conductivity for a wide range of various affecting parameters. This model is in good agreement with the considered experimental results.

\section{Nomenclature}

\section{Constant}

$C_{p_{f}} \quad$ Heat capacity per unit volume of the fluid, $\mathrm{kJ} / \mathrm{kg}$

$\mathrm{Cu} \quad$ Copper

d Diameter

Do Diffusion coefficient 
$E G \quad$ ethylene glycol

EMT Effective Medium Theory

$f \quad$ Factorial function

$h \quad$ Convective heat transfer

coefficient

$k \quad$ Thermal conductivity

$k_{B} \quad$ Boltzmann constant

$l \quad$ Mean free path

n Shape factor

Pr Prandtl number

$\mathrm{Re} \cdot$ Reynolds number

$K_{R} \quad$ Kapitza resistance

$T$ Temperature

Greek symbols

$\alpha \quad$ Thermal conductivity ratio

$\phi \quad$ Volume fraction (or volume concentration)

$\mu \quad$ Dynamic viscosity

$v \quad$ Kinematic viscosity

$\rho$. Density

Subscripts

bf Base fluid

cl Nanoparticle cluster

e Equivalent

eff Effective

f. Fluid

layer Interfacial layer (nanolayer)

p Particle

\section{References}

1. Clement Kleinstreuer, Yu Feng, 2011, Experimental and theoretical studies of nanofluid thermal conductivity enhancement: a review, Nanoscale Research Letters.

2.Das, S., Choi, U.S., Yu, W., Pradeep, T., 2008, Nanofluids: Science and technology, 1st ed. John Wiley \& Sons.

3. Xiang-Qi Wang, Arun S. Mujumdar, 2008, A review of nanofluids - part 1: theoretical and numerical investigations, Brazilian journal of chemical engineering, vol. 25, pp. 613630.

4. Maxwell, J. C., 1873, A Treatise on Electricity and Magnetism, Clarendon Press, Oxford.

5. Hamilton, R. L., and Crosser, O. K., 1962, Thermal Conductivity of Heterogeneous Two-Component Systems, Ind. Eng. Chem. Fund., 1(3), pp. 187-191.

6. Bruggeman, D. A. G., 1935, The Calculation of Various Physical Constants of Heterogeneous Substances. I. The Dielectric Constants and Conductivities of Mixtures Composed of Isotropic Substances, Ann. Phys., 416(7), pp. 636-664.

7. Q. Xue, W.-M. Xu, 2005, A model of thermal conductivity of nanofluids with interfacial shells, Materials Chemistry and Physics 90 (2-3) 298301.

8. Hasselman, D. P. H. and Johnson, L. F., Effective thermal conductivity of 
composites with interfacial thermal barrier resistance, Journal of Composite Materials, vol. 21 , no. 6 , pp.508-515, 1987.

9. Wang, X., Xu, X., and Choi, S. U. S., Thermal Conductivity of NanoparticleFluid Mixture, Joumal of Thermophysics and Heat Transfer, vol. 13, pp. 474-480, 1999.

10. Y. Xuan, Q. Li, W. Hu, 2003, Aggregation structure and thermal conductivity of nanofluids, AIChE Journal 49 (4) 1038-1043.

11. Seok Pil Jang and S. Choi, 2004, Role of Brownian Motion in the enhanced thermal conductivity of nanofluids, Applied physics letters, vol. 84, no 21.

12. Seok Pil Jang and S. Choi, 2007, Effects of Various Parameters on Nanofluid Thermal Conductivity, Journal of heat transfer, vol. 129.

13. Ravi Prasher, Prajesh Bhattacharya, Patrick E. Phelan, 2005, Thermal conductivity of nanoscale colloidal solutions (Nanofluids), Physics Review Letters, 94, 025901.

14. Junemoo Koo and Clement Kleinstreuer, 2004, A New Thermal Conductivity Model for Nanofluids, Journal of Nanoparticle research, (6), pp. 577-588.

15. V. Vasu, K. Rama Krishna and A C S Kumar, 2007, Analytical prediction of forced convective heat transfer of fluids embedded with nanostructured materials (nanofluids), Pramana journal of physics, vol. 69 , no.3, pp. 411-421.

16. Schwartz, L. M., Garboczi, E. J., and Bentz, D. P. 1995. Interfacial transport in porous media: Application to dc electrical conductivity of mortars, Journal of Applied Physics, 78(10), 5898-5908.

17. Das, S. K., Putra, N., Thiesen, P., and Roetzel, W., 2003. Temperature Dependence of Thermal Conductivity Enhancement for Nanofluids, Transactions of the ASME, Journal of Heat Transfer, vol. 125, pp. 567-574.

18. D. Hemanth Kumar, Hrishikesh E. Patel, V. R. Rajeev Kumar, T. Sundararajan, T. Pradeep, and Sarit K. Das, 2004, Model for Heat Conduction in Nanofluids, Physical Review Letters, vol. 93.

19. Xiang-Qi Wang, Arun S. Mujumdar, 2007, Heat Transfer characteristics of nanofluids: a review, International Journal of Thermal Sciences, vol. 46, pp. 1-19.

20. Chon, C. H., Kihm, K. D., Lee, S. P., and Choi, S. U. S., 2005, Empirical Correlation Finding the Role of Temperatıre and Particle Size for Nanofluid $\left(\mathrm{Al}_{2} \mathrm{O}_{3}\right)$ Thermal 
Conductivity Enhancement, Appl. Phys. L̇ett., 87(15), 153107.

21. A.R. Moghadassi, S. Masoud Hosseini, D. 'Henneke and A. Elkamel, 2009, A Model o: Nanofluids Effective Thermal Conductivity Based on Dinensionless Groups, Journal of thermal analysis and calorimetry, vol. 96.

22. Mostafa Keshavarz Morajevi, Hadi Hezavah and Reza Davarnejad, 2010, A New Model for the Thermal Conductivity Prediction of $\mathrm{Al} 2 \mathrm{O} 3$ and CuO Nanofluids in Water, World Applied Sciences Journal 9(10), pp: 1095-1099.

23. P.C. Mukesh Kumar, J. Kumar and S. Sendhilnathan, 2010, Theoretical model to determine the thermal conductivity of IJanofluids, International journal of engineering, science and technology, vol. 2(7)

24. Michael P. Beck, 2008, Thermal Conductivity of Metal Oxide Nanofluids, $\mathrm{PhD}$ thesis, Georgia Institute of Technology.

25. Honorine Angue Minsta, Gilles Roy, Cong Tam Nguyen, 2007, New Temperature Dependent Thermal Conductivity Data of Water Based Nanofluids, Proceedings of the 5th IASME/WSEAS Int. Conference on Heat Transfer, Thermal Engineering and Environment, Athens, Greece, August 25-27, 2007.

26. Hrishikesh E. Patel, T. Sundararajan, Sarit K. Das, 2010, An experimental investigation into the thermal conductivity enhancement in oxide and metallic nanofluids, Journal of Nanoparticle Research.

27. M. Izadi, S. Hossainpour and D. Galili-Vahid, 2008, Effects of Nanolayer Structure and Brownian Motion of Particles in Thermal Conductivity Enhancement of Nanofluids, proceedings of world academy of science, engineering and technology volume 31 . 